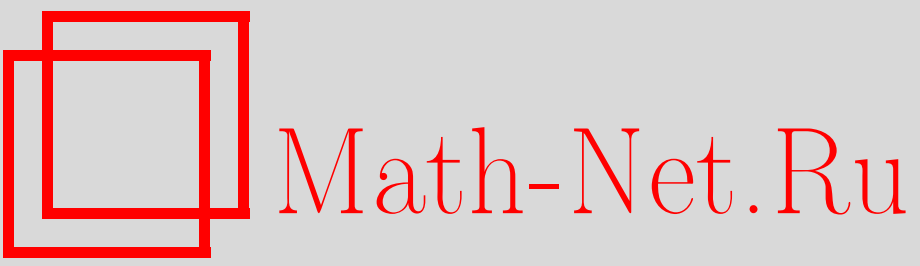

В. В. Белов, В. А. Максимов, Квазиклассические спектральные серии гелиеподобного атома в магнитном поле, ТМФ, 2001, том 126, номер 3, 455-474

DOI: https://doi.org/10.4213/tmf441

Использование Общероссийского математического портала Math-Net.Ru подразумевает, что вы прочитали и согласны с пользовательским соглашением

http://www.mathnet.ru/rus/agreement

Параметры загрузки:

IP : 54.210 .77 .194

26 апреля 2023 г., 15:23:04 


\author{
ТЕОРЕТИЧЕСКАЯ \\ И МАТЕМАТИЧЕСКАЯ \\ ФИЗИКА \\ Том 126, № 3 \\ март, 2001
}

\author{
(C) 2001 г. \\ В.В. Белов* , В. А. Максимов*
}

\title{
КВАЗИКЛАССИЧЕСКИЕ СПЕКТРАЛЬНЫЕ СЕРИИ ГЕЛИЕПОДОБНОГО АТОМА В МАГНИТНОМ ПОЛЕ
}

\begin{abstract}
Для модели двух электронов в поле неподвижного центра на основе комплексного метода ВКБ (теории комплексного ростка Маслова) построены квазиклассические спектральные серии собственных значений оператора Паули во внешнем магнитном поле с учетом спин-орбитального, спин-спинового взаимодействий и квадрупольного момента ядра. Эти серии отвечают найденному новому типу замкнутых фазовых траекторий - относительным положениям равновесия соответствующей классически неинтегрируемой системы. Получены явные эффективные формулы для тонкого (эффект Зеемана) и сверхтонкого расщеплений квазиклассических уровней энергии гелиеподобного иона с произвольным зарядом ядра $Z$ во всей области изменения величины магнитного поля, включая предельные случаи слабого и сверхсильного полей.
\end{abstract}

\section{1. ВВЕДЕНИЕ И ПОСТАНОВКА ЗАДАЧИ}

Работа посвяшена исследованию спектра гелиеподобных атомов (во внешнем магнитном поле) в рамках квазиклассического приближения. Как известно, квазиклассическое приближение реализует принцип соответствия результатов квантовой и классической теорий. В частности, в спектральных квантовых задачах квазиклассический подход позволяет сопоставить инвариантным объектам соответствующей классической системы приближенные собственные значения и собственные функции квантового гамильтониана. Такое сопоставление называют квазиклассическим квантованием.

Квазиклассическое квантование инвариантных (полномерных) лагранжевых торов в интегрируемых и близких к интегрируемым классических системах (квантование Эйнштейна-Бриллюэна-Келлера (ЭБК)) дает серии квазиклассических уровней энергии, а метод Вентцеля-Крамерса-Бриллюэна (ВКБ) - и соответствующие волновые функции одномерных квантовых гамильтонианов. Строгая математическая реализация этих методов в многомерных спектральных задачах, в том числе и с матричными гамильтонианами (многомерный метод ВКБ), была осушествлена в работах Маслова [1], [2] в его теории канонического оператора с вещественной фазой. Как отмечает в своей известной книге [3] Лере, квантование Маслова дает результат старой квантовой теории, сохраняя простоту вычислений; это квантование, возникаюшее из чисто математических рассмотрений, может заменить квантование Бора-Зоммерфельда. С физической точки

* Московский государственный институт электроники и математики, Москва, Россия. E-mail: belov@amath.msk.ru,pm@miem.edu.ru 
зрения такая замена будет оправданна, если она окажется применимой к атому с несколькими электронами.

Простейшей моделью такого атома является гелиеподобная система: два электрона в поле неподвижного центра. Соответствующая классическая система в этой ограниченной “заряженной” задаче трех тел является неинтегрируемой по Лиувиллю. Тем не менее и в случае неинтегрируемых гамильтоновых систем удается получить информацию о спектре соответствуюшей квантовой задачи на основе развитого относительно недавно в работах [4]-[6] комплексного метода ВКБ (теории комплексного ростка Маслова). Геометрические объекты, пригодные для квантования в этой теории, - это инвариантные маломерные лагранжевы торы $\Lambda^{k}(0 \leqslant k<n, n$-размерность конфигурационного пространства системы), обладаюшие дополнительным свойством орбитальной устойчивости (в линейном приближении). Явное (в аналитической форме) построение таких объектов для классически неинтегрируемых систем даже в случае $k=1$ ( $\Lambda^{1}$ - замкнутая фазовая траектория) является отдельной весьма нетривиальной задачей классической механики.

Заметим, что для атома гелия эта задача, как и проблема квантования его замкнутых фазовых орбит, имеет длинную историю. Еще в 1921 году в работе Ленгмюра [7] были вычислены орбиты, отвечаюшие планарному коррелированному движению двух электронов (в плоскости $z=0$ с общим угловым моментом $\mathbf{L}=0$ ), и получены квазиклассические уровни энергии по формулам старой квантовой теории Бора.

В настоящее время в физической литературе периодические коррелированные движения электронов в атоме гелия - предмет интенсивного (в основном численного) исследования (см., например, [8]-[10] и цитированную там литературу). Это связано с тем, что такие типы движения при условии их классической (линейной) стабильности порождают дважды возбужденные (с главными квантовыми числами $n \sim 10$ и выше $[11],[12])$ долгоживущие состояния квантовой системы, несмотря на то что их энергия выше, чем энергия одноэлектронной ионизации атома [13], [14]. Такие состояния наблюдались в экспериментах по мультифотонному лазерному возбуждению гелиеподобных атомов [15], где они проявлялись в виде узких резонансов, энергия которых чрезвычайно близка к энергии двойной ионизации атома. Лишь сравнительно недавно [16] была доказана стабильность ленгмюровских орбит, а также найдена устойчивая конфигурация коррелированного коллинеарного движения электронов (с общим угловым моментом $\mathbf{L}=0$ ), находяшихся по одну сторону от ядра, - так называемая $Z^{2+} e^{-} e^{-}$-конфигурация ("frozen planet configuration") [17]. На основе эвристической полуклассической теории Гутцвиллера [18] в работах [17], [9], [19]-[21] были вычислены тройные (с тремя квантовыми числами) ридберговские серии уровней энергии дважды возбужденных состояний: симметричных $\left(\left\langle r_{1}\right\rangle \sim\left\langle r_{2}\right\rangle\right)$ для ленгмюровских орбит и несимметричных $\left(\left\langle r_{1}\right\rangle \sim\left\langle r_{2}\right\rangle / 3\right)$ для $Z^{2+} e^{-} e^{-}$-конфигурации $(\langle r\rangle-$ радиальное среднее). Отметим, что именно последние состояния наблюдались в экспериментах по лазерному возбуждению атома бария [15].

В данной работе с помощью комплексного метода ВКБ получены аналитические эффективные формулы квазиклассических уровней энергии (спектральные серии) для 
дважды возбужденных состояний ${ }^{1)}$ гелиеподобных атомов, отвечающих новому типу коррелированного движения электронов (с общим орбитальным моментом $\mathbf{L} \neq 0$ ). Эти движения порождаются замкнутыми фазовыми траекториями - так называемыми относительными положениями равновесия (ОПР) соответствующей неинтегрируемой классической системы [22]. Они явно найдены в разделе 2 на основе известной процедуры редукции Марсдена-Вейнстейна [23].

В разделе 3 построены спектральные серии, качественно учитывающие как квадрупольный момент $\gamma \geqslant 0$ ядра атома, так и спиновые поправки, обусловленные спин-орбитальным и спин-спиновым взаимодействиями, и, как следствие, вычислены сверхтонкая и тонкая структуры квазиклассических уровней энергии скалярной квантовой задачи для гелиеподобных атомов. Заметим, что при $\gamma=0$ соответствующие квазиклассические волновые функции с точки зрения классификации, принятой в [24], это состояния двух эквивалентных электронов с орбитальными квантовыми числами $l_{1}=l_{2}=l / 2$, где $l$ - “коллективное" орбитальное квантовое число.

В разделе 4 мы квантуем в квазиклассическом приближении ОПР гелиеподобной системы, помешенной в однородное магнитное поле $B_{0}($ при $\gamma=0)$. Полученные формулы для зеемановского расщепления уровней энергии (как нормального, так и аномального с учетом диамагнетизма атома) справедливы для любых значений величины магнитного поля $B_{0}$, и что сушественно, включая промежуточную область полей $B_{0} \sim 10^{9}$ Гс, возникаюших на поверхности нейтронных звезд и белых карликов. Эта область наиболее трудна для анализа другими методами [25]-[27], поскольку кулоновское и магнитное взаимодействия в ней одного порядка. В этом же разделе рассмотрены предельные случаи слабого $\left(B_{0} \sim 10^{3} \Gamma \mathrm{c}\right)$ и сверхсильного $\left(B_{0} \sim 10^{11} \div 10^{13}\right.$ Гс $)$ магнитных полей и получены квазиклассические уровни энергии в виде разложения по степеням параметра, пропорционального полю $B_{0}$ (в случае слабых полей) и $B_{0}^{-1 / 2}$ (в случае сверхсильных полей). Последняя задача о поведении спектра гелиеподобных атомов в сверхсильном магнитном поле весьма актуальна в связи с недавними исследованиями мягкого рентгеновского излучения врашаюшихся пульсаров [28]-[30].

Подчеркнем еше раз, что нахождение ОПР является отдельной нетривиальной задачей в классической механике неинтегрируемых систем [22], [31]. Известно, что для систем с циклическими переменными ОПР определяются точками покоя эффективного гамильтониана на приведенном фазовом пространстве [22]. В более общем случае (когда циклических переменных нет или они явно не выделены) для гамильтоновых систем с непрерывной группой симметрий такие траектории могут быть получены с помощью известной классической процедуры редукции Марсдена-Вейнстейна [23]. В рассматриваемой ситуации классический гамильтониан гелиеподобной системы допускает непрерывные группы симметрий: группу $S O(2)$ при $B_{0} \neq 0$ и группу $S O(3)$ при $B_{0}=$ 0. В этих случаях ОПР гамильтоновой системы отвечают коррелированные движения электронов по равновесным орбитам действия групп $S O(2)$ и $S O(3)$ с полным орбитальным моментом электронов $\mathbf{L} \neq 0$ - окружностям радиусов $R_{1}$ и $R_{2}$, лежашим в плоскости $z=0$.

\footnotetext{
1) По-видимому, эти возбужденные долгоживущие состояния, так же как и состояния, отвечающие устойчивым конфигурациям ленгмюровских и "frozen planet" opбит, могут быть обнаружены в экспериментах по лазерному возбуждению гелиеподобных атомов.
} 
При отсутствии внешнего магнитного поля (диагональное гамильтоново действие группь $S O(3)$ на пространстве $\mathbb{R}^{12}=\mathbb{R}_{\mathbf{p}}^{6} \times \mathbb{R}_{\mathbf{q}}^{6}$ ) процедура редукции позволила дать полную классификацию всех относительных положений равновесия гелиеподобной системы с учетом квадрупольного момента атома и исследовать их (орбитальную) устойчивость в широкой области параметров классической системы ${ }^{2}$. . Оказывается, что структура фазового пространства гелиеподобного атома, и в частности структура его ОПР, весьма чувствительна к малым возмущениям гамильтониана, связанным с квадрупольным моментом ядра $\gamma, \gamma \sim 10^{-25} \mathrm{~cm}^{2}$. Так, например, в случае $\gamma=0$ у классической системы, состояшей из двух электронов в поле неподвижного центра, сушествует лишь единственное семейство симметричных ОПР, для которых радиусы орбит электронов $R_{1}$ и $R_{2}$ совпадают. При учете квадрупольного момента ядра $(\gamma>0)$ помимо этого симметричного ОПР в системе возникают еше два семейства несимметричных ОПР с энергией, близкой к энергии ионизации атома гелия (см. раздел 3 ), в каждом из которых радиусы равновесных орбит двух электронов различны $\left(R_{1}(\gamma) \neq R_{2}(\gamma)\right)$.

Перейдем к точной постановке задачи. Рассмотрим следуюшую спектральную задачy:

$$
\begin{gathered}
\widehat{H} \Psi=E \Psi, \quad \Psi \in \mathbb{L}_{2}\left(\mathbb{R}_{\mathbf{q}_{1}, \mathbf{q}_{2}}^{6}\right) \times \mathbb{C}^{2} \times \mathbb{C}^{2}, \\
\Psi={ }^{t}\left(\Psi_{1}, \Psi_{2}\right), \quad \Psi_{i}={ }^{t}\left(\Psi_{i 1}, \Psi_{i 2}\right), \quad i=1,2,
\end{gathered}
$$

с квантовым гамильтонианом Паули $\widehat{H}$ вида [32]

$$
\begin{gathered}
\widehat{H}=\widehat{H}_{0}+\hbar \widehat{H}_{\mathrm{s}-\mathrm{f}}+\hbar^{2} \widehat{H}_{\mathrm{s}-\mathrm{s}}, \\
\widehat{H}_{0}=\frac{1}{2 m_{e}}\left(-i \hbar \nabla_{\mathbf{q}_{1}}+\frac{e_{0}}{c} \mathbf{A}_{1}\right)^{2}+\frac{1}{2 m_{e}}\left(-i \hbar \nabla_{\mathbf{q}_{2}}+\frac{e_{0}}{c} \mathbf{A}_{2}\right)^{2}+V_{\gamma}\left(\mathbf{q}_{1}, \mathbf{q}_{2}\right), \\
V_{\gamma}=-\frac{Z e_{0}^{2}}{r_{1}}-\frac{Z e_{0}^{2}}{r_{2}}+\frac{e_{0}^{2}}{r_{12}}+e_{0}^{2} \gamma\left(\frac{1}{r_{1}^{3}}+\frac{1}{r_{2}^{3}}\right), \quad \gamma \geqslant 0, \\
\hbar \widehat{H}_{\mathrm{s}-\mathrm{f}}=\frac{e_{0} \hbar}{2 m_{e} c}\left(\mathcal{H}_{1} \sigma_{1}+\mathcal{H}_{2} \sigma_{2}\right)+ \\
+\frac{e_{0} \hbar}{4 m_{e}^{2} c^{2}}\left\{\left[\mathcal{E}_{1} \times\left(-i \hbar \nabla_{\mathbf{q}_{1}}\right)+\frac{2 e_{0}}{r_{12}^{3}} \mathbf{r}_{12} \times\left(-i \hbar \nabla_{\mathbf{q}_{2}}\right)\right] \sigma_{1}+\right. \\
\left.+\left[\mathcal{E}_{2} \times\left(-i \hbar \nabla_{\mathbf{q}_{2}}\right)+\frac{2 e_{0}}{r_{12}^{3}} \mathbf{r}_{21} \times\left(-i \hbar \nabla_{\mathbf{q}_{1}}\right)\right] \sigma_{2}\right\} \\
\hbar^{2} \widehat{H}_{\mathrm{s}-\mathrm{s}}=\frac{e_{0}^{2} \hbar^{2}}{4 m_{e}^{2} c^{2}} \frac{1}{r_{12}^{3}}\left[\sigma_{1} \sigma_{2}-\frac{3\left(\sigma_{1} \mathbf{r}_{12}\right)\left(\sigma_{2} \mathbf{r}_{12}\right)}{r_{12}^{2}}\right] .
\end{gathered}
$$

Здесь $V_{\gamma}\left(\mathbf{q}_{1}, \mathbf{q}_{2}\right), \mathbf{q}_{i}=\left(x_{i}, y_{i}, z_{i}\right), i=1,2$, есть потенциальная энергия кулоновского взаимодействия между двумя электронами $\left(e_{0}=-e, e, m_{e}-\right.$ заряд и масса электрона) и неподвижным ядром гелиеподобного атома с зарядом $Z$, помешенным в начало координат; расстояния между электронами и ядром обозначены через $r_{i}, i=1,2$, между электронами - через $r_{12}$. Последнее слагаемое в $V_{\gamma}$ при определенных условиях [24] описывает квадрупольное взаимодействие нуклонов ядра с электронами. В

\footnotetext{
2)Этот вспомогательный с точки зрения квазиклассических асимптотик квантовой задачи результат представляет, на наш взгляд, самостоятельный интерес для известной классической ограниченной "заряженной” задачи трех тел при исследовании структуры ее фазовых траекторий.
} 
операторе взаимодействия спинов электронов с внешним однородным магнитным полем $\mathbf{A}_{i}=B_{0}\left(-y_{i}, x_{i}, 0\right) / 2, B_{0}=$ const, $i=1,2$, матрицы Паули $\sigma_{i}=\left(\sigma_{i}^{1}, \sigma_{i}^{2}, \sigma_{i}^{3}\right)$ действуют на $i$-ю спинорную компоненту волновой функции $\Psi, \mathcal{E}_{i}=-\nabla_{\mathbf{q}_{i}} V_{\gamma}\left(\mathbf{q}_{1}, \mathbf{q}_{2}\right) / e_{0}, \mathcal{H}_{i}=$ $\nabla_{\mathbf{q}_{i}} \times \mathbf{A}_{i}, \hbar^{2} \widehat{H}_{\mathrm{s}-\mathrm{s}}-$ оператор взаимодействия спиновых магнитных дипольных моментов электронов.

Комплексньй метод ВКБ позволяет сопоставить квазиклассические $(\hbar \rightarrow 0)$ серии собственных функций $\Psi_{\vec{n}, \xi}(\mathbf{q}, \hbar)$ и собственных значений $E_{\vec{n}, \xi}(\hbar)$ задачи $(1),(2)$ - квазимод оператора $\widehat{H}$, замкнутым фазовым траекториям $\Lambda^{1}$ соответствующей классически неинтегрируемой системы Гамильтона

$$
\begin{gathered}
\dot{\mathbf{p}}=-H_{\mathbf{q}}, \quad \dot{\mathbf{q}}=H_{\mathbf{p}}, \\
(\mathbf{p}, \mathbf{q}) \in \mathbb{R}_{\mathbf{p}}^{6} \times \mathbb{R}_{\mathbf{q}}^{6}, \quad \mathbf{q}={ }^{t}\left(\mathbf{q}_{1}, \mathbf{q}_{2}\right), \quad \mathbf{p}={ }^{t}\left(\mathbf{p}_{1}, \mathbf{p}_{2}\right), \\
H(\mathbf{p}, \mathbf{q})=\frac{1}{2 m_{e} c} \sum_{i=1}^{2} \mathbf{p}_{i}^{2}+V_{\gamma}(\mathbf{q})+\frac{e_{0} B_{0}}{2 m_{e}} L_{z}+\frac{e_{0}^{2} B_{0}^{2}}{8 m_{e} c^{2}}\left(x_{1}^{2}+y_{1}^{2}+x_{2}^{2}+y_{2}^{2}\right),
\end{gathered}
$$

где $L_{z}$ - проекция полного момента двух электронов $\mathbf{L}=\mathbf{q}_{1} \times \mathbf{p}_{1}+\mathbf{q}_{2} \times \mathbf{p}_{2}$ на направление магнитного поля (ось $z$ ). Здесь $\vec{n}$ - полньй набор орбитальных квантовых чисел двух электронов: $\vec{n}=\left(\vec{n}_{1}, \vec{n}_{2}\right), n_{i} \in \mathbb{Z}^{3}$, а $\xi$ - спиновое квантовое число $(\xi= \pm 1,0 ; 0)$ нумерует четыре спиновых состояния системы двух электронов, разделенных по значению полного спина $S$ и его проекции $m_{s}$ на ось $z$. А именно, значения $\xi= \pm 1,0$ отвечают трем состояниям $\left(S=1, m_{s}= \pm 1,0\right)$, а значение $\xi=0$ - состоянию $\left(S=0, m_{s}=0\right)$.

Напомним определение квазимод задачи $(1),(2)$, отвечающих замкнутым фазовым траекториям $\Lambda^{1}$ классической системы $(3),(4)$. Пусть фазовая траектория $\Lambda^{1}\left(E_{0}\right)$ принадлежит гладкому однопараметрическому семейству фазовых кривых $\Lambda^{1}(E)$ в $\mathbb{R}^{12}$, где параметр $E \in\left(E_{1}, E_{2}\right)$ - классическая энергия системы. Последовательность значений $E_{\vec{n}, \xi}=E_{\vec{n}, \xi}(\hbar)$ (где орбитальные квантовые числа $\vec{n}$, вообше говоря, могут зависеть от $\hbar)$ и функций $\Psi_{E_{\vec{n}, \xi}(\hbar)}(\mathbf{q}, \hbar) \in \mathbb{L}_{2}\left(\mathbb{R}_{\mathbf{q}}^{6}\right) \times \mathbb{C}^{2} \times \mathbb{C}^{2}, \hbar \in(0,1]$, называется квазиклассической спектральной серией (квазимодой квантового гамильтониана $\widehat{H}$ ), отвечаюшей классической траектории $\Lambda^{1}\left(E_{0}\right)$, если выполнены следуюшие условия:

1) $\lim _{\hbar \rightarrow 0} E_{\vec{n}, \xi}(\hbar)=\left.H(\mathbf{p}, \mathbf{q})\right|_{\Lambda^{k}\left(E_{0}\right)}$ (соответствие между квазиклассической спектральной серией $E_{\vec{n}, \xi}(\hbar)$ и классическим движением на уровне энергии $\left.E_{0}\right)$;

2) $\Psi_{E_{\vec{n}, \xi}(\hbar)}(\mathbf{q}, \hbar)=O(1)$ для почти всех ${ }^{3)} \mathbf{q} \in \pi_{\mathbf{q}}\left(\Lambda^{1}\left(E_{0}\right)\right)$, где $\pi_{\mathbf{q}}\left(\Lambda^{1}\left(E_{0}\right)\right)$ - проекция $\Lambda^{1}\left(E_{0}\right)$ на конфигурационное пространство $\mathbb{R}_{\mathbf{q}}^{6}$ (соответствие между квазиклассической серией асимптотических собственных функций и $\left.\Lambda^{1}\left(E_{0}\right)\right)$;

3) $\left\|\left(\widehat{H}-E_{\vec{n}, \xi}(\hbar)\right) \Psi_{E_{\vec{n}, \xi}(\hbar)}(\mathbf{q}, \hbar)\right\|_{\mathbb{L}_{2}\left(\mathbb{R}_{\mathbf{q}}^{6}\right) \times \mathbb{C}^{2} \times \mathbb{C}^{2}}=O\left(\hbar^{N}\right), N>1$ (условие формальной асимптотики).

Опишем кратко, следуя работам [5], [6], структуру квазимод, возникаюших при квазиклассическом квантовании методом комплексного ростка Маслова при отсутствии фокальных точек на $\Lambda^{1}:|\dot{Q}| \neq 0$, семейства замкнутых фазовых траекторий классической системы $\Lambda^{1}(E)=\{\mathbf{p}=P(t), \mathbf{q}=Q(t)\}$, где $P(t), Q(t)$ являются периодическими

\footnotetext{
3) Исключение составляют фокальные точки или точки, принадлежащие каустическим множествам.
} 
функциями с периодом $T=T(E)$. В квазиклассическом приближении $(\hbar \rightarrow 0)$ для задачи $(1),(2)$ орбитальные $\left(\mathbf{q}=\left(\mathbf{q}_{1}, \mathbf{q}_{2}\right)\right)$ и спиновые переменные могут быть разделены с точностью $O\left(\hbar^{2}\right)$. А именно, квазиклассические волновые функции матричного оператора $\widehat{H}$ имеют следующую структуру:

$$
\Psi_{\vec{n}, \xi}(\mathbf{q}, \hbar)=\Psi_{\vec{n}}(\mathbf{q}, \hbar) \mathbf{f}_{\xi}(\mathbf{q})
$$

а соответствующий квазиклассический спектр имеет вид

$$
E_{\vec{n}, \xi}(\hbar)=E_{\vec{n}}(\hbar)+\hbar \mu_{\xi}+O\left(\hbar^{2}\right) .
$$

Здесь $\left(E_{\vec{n}}(\hbar), \Psi_{\vec{n}}(\mathbf{q}, \hbar)\right)$ - квазимоды скалярного оператора Шредингера $\widehat{H}_{0}$, отвечающие $\Lambda^{1}$ :

$$
\widehat{H}_{0} \Psi_{\vec{n}}(\mathbf{q}, \hbar)=E_{\vec{n}}(\hbar) \Psi_{\vec{n}}(\mathbf{q}, \hbar)+O\left(\hbar^{\frac{3}{2}}\right), \quad \Psi_{\vec{n}}(\mathbf{q}, \hbar) \in \mathbb{L}_{2}\left(\mathbb{R}_{\mathbf{q}}^{6}\right)
$$

где, так же как и в условии 3$)$, под $O\left(\hbar^{3 / 2}\right)$ понимается оценка невязки уравнения в среднем - по норме $\mathbb{L}_{2}$. Спиновые поправки к спектру $\mu_{\xi}$ и соответствующий спинор $\mathbf{f}_{\xi}(\mathbf{q})$ находятся как решения спектральной задачи для уравнения поляризации на фазовой кривой $\Lambda^{1}$ :

$$
-i \frac{d}{d t} \mathbf{f}_{\xi}+\left.\Pi\right|_{\Lambda^{1}} \mathbf{f}_{\xi}=\mu_{\xi} \mathbf{f}_{\xi}, \quad \mathbf{f}_{\xi}: \Lambda^{1} \rightarrow \mathbb{C}^{2} \times \mathbb{C}^{2}, \quad \mu_{\xi} \in \mathbb{R}
$$

Здесь $\mathbf{f}_{\xi}=\mathbf{f}_{\xi}(\mathbf{q}=Q(t))$ и $d / d t$ есть дифференцирование вдоль траекторий классической системы (3), а матрица поляризации П имеет вид (см., например, [6], [33])

$$
\begin{aligned}
\Pi= & \frac{e_{0}}{2 m_{e} c}\left(\mathcal{H}_{1} \sigma_{1}+\mathcal{H}_{2} \sigma_{2}\right)+\frac{e_{0}}{4 m_{e}^{2} c^{2}}\left\{\left(\mathcal{E}_{1} \times \mathbf{p}_{1}+\frac{2 e_{0}}{r_{12}^{3}} \mathbf{r}_{12} \times \mathbf{p}_{2}\right) \sigma_{1}+\right. \\
& \left.+\left(\mathcal{E}_{2} \times \mathbf{p}_{2}+\frac{2 e_{0}}{r_{12}^{3}} \mathbf{r}_{21} \times \mathbf{p}_{1}\right) \sigma_{2}\right\}+\frac{e_{0}^{2} \hbar}{2 m_{e}^{2} c^{2}} \frac{1}{r_{12}^{3}}\left\{\sigma_{1} \sigma_{2}-\frac{3\left(\sigma_{1} \mathbf{r}_{12}\right)\left(\sigma_{2} \mathbf{r}_{12}\right)}{r_{12}^{2}}\right\} .
\end{aligned}
$$

Параметр $\xi= \pm 1,0,0$ нумерует четыре спиновых состояния системы двух электронов, разделенных по значению полного спина $\mathbf{s}$ и его проекции $m_{s}$ на ось $z$. А именно, значения $\xi= \pm 1,0$ отвечают трем состояниям $\left(\mathbf{s}=1, m_{s}= \pm 1,0\right)$, а значение $\xi=0$ - состоянию $\left(\mathbf{s}=0, m_{s}=0\right)$.

ЗАмечАниЕ 1. Используя стандартную технику [1], несложно показать, что в силу оценки 3 в определении квазимод асимптотические собственные значения $E_{\vec{n}, \xi}(\hbar)(5)$ отстоят от точных собственных значений $E^{\text {ех }}$ задачи $(1),(2)$ на величину порядка $O\left(\hbar^{3 / 2}\right)$, т.е. сушествует значение $E^{\mathrm{ex}}$ такое, что $\left|E_{\vec{n}, \xi}(\hbar)-E^{\mathrm{ex}}\right| \leqslant C \hbar^{3 / 2} \quad(C>0$ - константа). При этом асимптотические собственные функции $\Psi_{\vec{n}, \xi}(\mathbf{q}, \hbar)$ близки к некоторой линейной комбинации точных собственных функций.

ЗАмЕчАниЕ 2. Формальная процедура вычисления асимптотик по $\hbar(\hbar \rightarrow 0)$ подразумевает, что в рассматриваемой ситуации безразмерным параметром квазиклассических разложений является отношение длины волны де Бройля $\lambda$ к характерным размерам системы $R: \lambda / R \sim 1 / l$, где $l$ - "коллективное" орбитальное квантовое число двух электронов. Другими двумя параметрами разложения здесь являются отношения $\sqrt{\nu_{1} / l}$ и 
$\sqrt{\nu_{2} / l} \sim \sqrt{\hbar} \ll 1$, где $\nu_{1}, \nu_{2}-$ номера мод радиальных осцилляций электронов около их равновесной орбиты $\pi_{\mathbf{q}} \Lambda^{1}(E)$, лежащей в плоскости $z=0$.

Отметим, что результаты работы, касающиеся квазиклассического спектра скалярной задачи (6), носят полуэвристический характер. Мы не указьваем асимптотических собственных функций, а приводим формулы лиш для части квазиклассического спектра задачи (6), соответствуюшего устойчивым в линейном приближении степеням свободы в окрестности $\Lambda^{1}$, а именно, устойчивым малым радиальным колебаниям около равновесной орбиты $\pi_{q} \Lambda^{1}$. Отвечаюшие таким движениям Флоке решения системы в вариациях (линеаризации системы (3) в окрестности $\Lambda^{1}$ ) имеют нетривиальные мультипликаторы - собственные значения матрищы монодромии [34] $\lambda_{1,2}=e^{ \pm i \beta_{1}}$ и $\lambda_{3,4}=e^{ \pm i \beta_{2}}$, где $\beta_{j} \in \mathbb{R}$ и $\beta_{j} \neq 0 \bmod 2 \pi$. Остальные собственные значения $\lambda_{i+4}, i=1,2,3,4$ (кратности два) равны 1 (это связано с наличием у классической системы (3) четырех первых интегралов $H_{0}, \mathbf{L}_{z}, \mathbf{L}_{x}$ и $|\mathbf{L}|^{2}$, находящихся в инволюции на плоскости $\mathbf{L}_{x}=\mathbf{L}_{y}=0$ $\left(\mathbf{L}_{z} \neq 0\right)$, содержащей ОПР этой системы). Можно показать, что приведенная матрица монодромии [34] диагонализуема. Это означает, что комплексный росток $r^{6}$ над кривой $\Lambda^{1}$ является не единственным [35]. Здесь мы сталкиваемся с ситуацией, аналогичной той, которая изучалась для квантовой анизотропной задачи Кеплера [36] и трехмерного ангармонического осциллятора [37]. С точки зрения теории квазиклассических асимптотик эта резонансная ситуация приводит к асимптотическому вырождению спектра скалярной задачи (6) (в пределах точности $O\left(\hbar^{3 / 2}\right)$ ). На основе результатов работ [36]-[38] это асимптотическое вырождение спектра в принципе можно снять в следующих порядках по $\hbar \rightarrow 0\left(\bmod O^{5 / 2}\right)$, построив на кривой $\Lambda^{1}$ “нелинейньй" росток, учитывающий как кубические, так и четвертые степени разложения функции Гамильтона $H$ в окрестности $\Lambda^{1}$ по ростковым переменным. Решение такой задачи требует дополнительных конструкций и составляет тему отдельной работы.

\section{2. РЕДУКЦИЯ ПО ДЕЙСТВИЮ ГРУППЫ $S O(3)$ И ПОСТРОЕНИЕ ИНВАРИАНТНОГО КОМПЛЕКСНОГО РОСТКА}

При отсутствии внешнего магнитного поля $\left(B_{0}=0\right)$ инвариантность гамильтониана $H(\mathbf{p}, \mathbf{q})(4)$ относительно действия группы $G=S O(3)$ порождает диагональное действие $G$ на евклидовом фазовом пространстве $\mathbb{R}_{\mathbf{p}, \mathbf{q}}^{12}=\mathbb{R}_{\mathbf{p}}^{6} \times \mathbb{R}_{\mathbf{q}}^{6}$ со стандартной симплектической структурой $\omega=d p \wedge d q$. Гамильтоново действие $G$ на фазовом пространстве определяет отображение $\mathbb{R}^{12}$ в дуальное пространство алгебры Ли группы - отображение момента:

$$
\mathcal{L}: \mathbb{R}^{12} \rightarrow g^{\star} \approx \mathbb{R}^{3}, \quad \mathcal{L}=\mathbf{q}_{1} \times \mathbf{p}_{1}+\mathbf{q}_{2} \times \mathbf{p}_{2}
$$

Следуя [22], введем редуцированное (по диагональному действию группы $G$ ) фазовое пространство $B_{L}=M_{L} / G_{L}$, где $M_{L}=\mathcal{L}^{-1}(L), L \in g^{\star}$ - множество уровня отображения момента, $G_{L}=\left\{g \in G: \operatorname{Ad}_{g}^{\star} L=L, L \in g^{\star}\right\}=S O(2)$ - стационарная подгруппа точки $L$ относительно коприсоединенного представления $G$. Для описания структуры редуцированного фазового пространства рассмотрим алгебру Ли $G_{\mu}$, состоящую из функций на $\mathbb{R}_{\mathbf{p}, \mathbf{q}}^{12}$, находящихся в инволюции с компонентами отображения момента относительно стандартной скобки Пуассона. Реализуем эту алгебру с помощью следующего 
набора координатных функций $\mu$ :

$$
\begin{aligned}
\mu=\left\{\mu_{1}\right. & =\frac{\left|\mathbf{p}_{1}\right|^{2}}{2}, \quad \mu_{2}=\frac{\left|\mathbf{q}_{1}\right|^{2}}{2}, \quad \mu_{3}=\left\langle\mathbf{p}_{1}, \mathbf{q}_{1}\right\rangle, \quad \mu_{4}=\frac{\left|\mathbf{p}_{2}\right|^{2}}{2}, \quad \mu_{5}=\frac{\left|\mathbf{q}_{2}\right|^{2}}{2}, \\
\mu_{6} & \left.=\left\langle\mathbf{p}_{2}, \mathbf{q}_{2}\right\rangle, \quad \mu_{7}=\left\langle\mathbf{q}_{1}, \mathbf{q}_{2}\right\rangle, \quad \mu_{8}=\left\langle\mathbf{p}_{1}, \mathbf{p}_{2}\right\rangle, \quad \mu_{9}=\left\langle\mathbf{p}_{1}, \mathbf{q}_{2}\right\rangle, \quad \mu_{10}=\left\langle\mathbf{p}_{2}, \mathbf{q}_{1}\right\rangle\right\} .
\end{aligned}
$$

Скобка Ли-Пуассона на дуальном пространстве $g_{\mu}^{\star} \approx \mathbb{R}_{\mu}^{10}\left(\mu=\left(\mu_{1}, \ldots, \mu_{10}\right)\right.$ - координаты на $\mathbb{R}_{\mu}^{10}$ ) алгебры Ли $G_{\mu}$ является вырожденной и имеет ранг 8 . Соответствуюшие функции Казимира $I_{l}(\mu), l=1,2$, этой алгебры вычисляются и имеют вид

$$
\begin{gathered}
I_{1}(\mu)=\mu_{7} \mu_{8}-\mu_{9} \mu_{10}+2 \mu_{1} \mu_{2}-\frac{\mu_{3}^{2}}{2}+2 \mu_{4} \mu_{5}-\frac{\mu_{6}^{2}}{2} \\
I_{2}(\mu)=\mu_{1} \mu_{2}\left(4 \mu_{4} \mu_{5}-\mu_{6}^{2}\right)-\mu_{5} \mu_{6} \mu_{3}^{2}+\frac{\mu_{3}^{2} \mu_{6}^{2}}{4}-\mu_{2} \mu_{5} \mu_{8}^{2}-\frac{\mu_{3} \mu_{6} \mu_{7} \mu_{8}}{2}-\mu_{1} \mu_{4} \mu_{7}^{2}+ \\
+\mu_{2} \mu_{6} \mu_{8} \mu_{9}+\mu_{3} \mu_{4} \mu_{7} \mu_{9}-\mu_{2} \mu_{4} \mu_{9}^{2}+\mu_{3} \mu_{5} \mu_{8} \mu_{10}+\mu_{1} \mu_{6} \mu_{7} \mu_{10}- \\
-\frac{\mu_{3} \mu_{6} \mu_{9} \mu_{10}}{2}-\mu_{1} \mu_{5} \mu_{10}^{2}+\left[\frac{\mu_{7} \mu_{8}}{2}-\frac{\mu_{9} \mu_{10}}{2}\right]^{2} .
\end{gathered}
$$

УТВЕРЖДЕНИЕ 1. Динамическая гамильтонова система на редуцированном фазовом пространстве $B_{L}$ имеет вид

$$
\begin{gathered}
\frac{d \mu_{i}}{d t}=\left\{H_{0}, \mu_{i}\right\}=\left[\Psi(\mu) \nabla_{\mu} H_{0}\right]_{i}, \quad i=1, \ldots, 10 \\
I_{1}(\mu)=\frac{L^{2}}{2}, \quad L \in \mathbb{R}^{+} \\
I_{2}(\mu)=0
\end{gathered}
$$

әде функиия Гамильтона

$$
\begin{aligned}
& H_{0}=H_{0}(\mu)=\frac{1}{m}\left(\mu_{1}+\mu_{4}\right)-\frac{Z e_{0}^{2}}{\sqrt{2 \mu_{2}}}-\frac{Z e_{0}^{2}}{\sqrt{2 \mu_{5}}}+ \\
& \quad+\frac{e_{0}^{2}}{\sqrt{2\left(\mu_{2}+\mu_{5}-\mu_{7}\right)}}+e_{0}^{2} \gamma\left(\left(2 \mu_{2}\right)^{-\frac{3}{2}}+\left(2 \mu_{5}\right)^{-\frac{3}{2}}\right)
\end{aligned}
$$

а кососимметрическая $(10 \times 10)$-матрица

$$
\Psi(\mu)=\left(\begin{array}{cccccccccc}
0 & \mu_{3} & -2 \mu_{1} & 0 & 0 & 0 & 0 & \mu_{9} & 0 & \mu_{8} \\
-\mu_{3} & 0 & -2 \mu_{2} & 0 & 0 & 0 & -\mu_{10} & 0 & -\mu_{7} & 0 \\
-2 \mu_{1} & 2 \mu_{2} & 0 & 0 & 0 & 0 & -\mu_{8} & \mu_{7} & -\mu_{9} & \mu_{10} \\
0 & 0 & 0 & 0 & \mu_{6} & 2 \mu_{4} & 0 & \mu_{10} & \mu_{8} & 0 \\
0 & 0 & 0 & -\mu_{6} & 0 & -2 \mu_{5} & -\mu_{9} & 0 & 0 & -\mu_{7} \\
0 & 0 & 0 & -2 \mu_{4} & 2 \mu_{5} & 0 & -\mu_{8} & \mu_{7} & \mu_{9} & -\mu_{10} \\
0 & \mu_{10} & \mu_{8} & 0 & \mu_{9} & \mu_{8} & 0 & \mu_{3}+\mu_{6} & 2 \mu_{1} & 2 \mu_{4} \\
-\mu_{9} & 0 & -\mu_{7} & -\mu_{10} & 0 & -\mu_{7} & -\mu_{3}-\mu_{6} & 0 & -2 \mu_{5} & -2 \mu_{2} \\
0 & \mu_{7} & \mu_{9} & -\mu_{8} & 0 & -\mu_{9} & -2 \mu_{4} & 2 \mu_{5} & 0 & \mu_{6}-\mu_{3} \\
-\mu_{8} & 0 & -\mu_{10} & 0 & \mu_{7} & \mu_{10} & -2 \mu_{4} & 2 \mu_{2} & \mu_{3}-\mu_{6} & 0
\end{array}\right) .
$$


Уравнение (9) есть уравнение Эйлера-Пуанкаре на $\mathbb{R}_{\mu}^{10}$, а формулы (10), (11) определяют однопараметрическое семейство $\Omega=\Omega(L), L \in \mathbb{R}^{+}$, орбит коприсоединенного представления в $\mathbb{R}_{\mu}^{10}$. Каждая из орбит семейства инвариантна относительно пуассонова фазового потока $g_{H_{0}}^{t}: \mathbb{R}_{\mu}^{10} \rightarrow \mathbb{R}_{\mu}^{10}$ и является четырехмерным симплектическим многообразием с невырожденной замкнутой 2-формой Кириллова [39]

$$
\omega_{\Omega}=\frac{1}{2} d \mu \wedge \Psi^{-1}(\mu) d \mu .
$$

На орбите $\Omega(L)$ рассмотрим класс нульмерных подмногообразий $\Lambda^{0}=\mu_{0}(L) \subset \Omega(L)$, аннулируюших форму $(12)\left(\left.\omega_{\Omega}\right|_{\mu_{0}(L)}=0\right)$ - точки покоя уравнения $(9)$,

$$
\Psi\left(\mu_{0}(L)\right) \nabla_{\mu} H_{0}\left(\mu_{0}(L)\right)=0 .
$$

Построим инвариантный комплексный росток в точке $\mu_{0}(L)$ - положительную лагранжеву плоскость $r\left(\mu_{0}(L)\right)$ в комплексифишированном касательном пространстве $\mathbf{C}_{T}(\Omega)_{\mu_{0}(L)} \quad\left(\operatorname{dim} r\left(\mu_{0}(L)\right)=\operatorname{dim} \Omega / 2=2\right) \quad$ (см. подробнее в [4], [5]). Хорошо известно [4], что необходимым и достаточным условием существования ростка является устойчивость точки покоя $\mu_{0}(L)$ в линейном приближении. А именно, все решения линеаризованного в окрестности $\mu_{0}(L)$ уравнения Эйлера-Пуанкаре

$$
\frac{d \mathbf{e}}{d t}=\mathcal{H}_{\mathrm{var}}(\mu) \mathbf{e}, \quad \mathbf{e}(t) \in \mathbb{C}^{10},
$$

ограничены при $t \in \mathbb{R}^{1}$. Здесь $\mathcal{H}_{\mathrm{var}}(\mu)$-постоянная матрица с элементами

$$
\left(\mathcal{H}_{\mathrm{var}}(\mu)\right)_{j s}=\left[\sum_{k=1}^{10}\left(\lambda_{j k}^{s} \frac{\partial H_{0}}{\partial \mu_{k}}+\Psi_{j k}(\mu) \frac{\partial^{2} H_{0}}{\partial \mu_{k} \partial \mu_{s}}\right)\right]_{\mu_{0}(L)},
$$

$j, s=1, \ldots, 10$, а $\lambda_{j k}^{s}$ - структурные константы алгебры Ли $G_{\mu} \quad\left(\left\{\mu_{j}, \mu_{k}\right\}=\right.$ $\left.\sum_{s=1}^{10} \lambda_{j k}^{s} \mu_{s}\right)$.

УТВЕРЖДЕНИЕ 2. Пусть точка покоя $\mu_{0}(L)$ устойчива в линейном приближении. Тогда ненулевые собственные значения матрицы $\mathcal{H}_{\mathrm{var}}(14)$ (показатели Флоке) чисто мнимье и имеют вид $\pm i \beta_{1}(L), \pm i \beta_{2}(L), \beta_{1,2} \in \mathbb{R}^{1}$. Существуют два линейно независимых решения уравнения (13), $\mathbf{e}^{1}(t), \mathbf{e}^{2}(t) \in \mathbf{C}^{\mathbf{C}} T(\Omega)_{\mu_{0}(L)}, \quad \mathbf{e}^{j}(t)=$ $e^{i \beta_{j} t} \mathbf{e}^{j}(0)$, удовлетворяющих условиям

$$
\omega_{\Omega}\left(\mathbf{e}^{j}(t), \mathbf{e}^{k}(t)\right)=0, \quad \omega_{\Omega}\left(\mathbf{e}^{j}(t), \overline{\mathbf{e}}^{k}(t)\right)=2 i \delta_{j k}, \quad j, k=1,2 .
$$

Указаннье решения $\mathbf{e}^{j}(t), j=1,2$, образуют базис на инвариантном комплексном ростке $r\left(\mu_{0}(L)\right)$.

Приведем теперь явные формулы для соответствуюшей замкнутой кривой $\Lambda^{1}$ в исходном фазовом пространстве $\mathbb{R}_{\mathbf{p}, \mathbf{q}}^{12}$, используя стандартную процедуру реконструкции [23] 
Обозначим через $\mu_{0}^{\alpha}(L), \alpha=1,2, \ldots, N, N=N(\gamma, L)$, точки покоя уравнения $(9)$, лежашие на орбите $\Omega(L)$. ОПР - решения классической системы $(3)$, отвечающие $\mu_{0}^{\alpha}(L)$, имеют вид

$$
\Lambda_{\gamma}^{\alpha}(L)=\left\{\begin{array}{c}
\mathbf{q}_{1}^{\alpha}=\left(\begin{array}{c}
R_{1}^{\alpha} \cos \Omega^{\alpha} t \\
R_{1}^{\alpha} \sin \Omega^{\alpha} t \\
0
\end{array}\right), \quad \mathbf{q}_{2}^{\alpha}=\left(\begin{array}{c}
-R_{2}^{\alpha} \cos \Omega^{\alpha} t \\
-R_{2}^{\alpha} \sin \Omega^{\alpha} t \\
0
\end{array}\right) \\
\mathbf{p}_{1}^{\alpha}=\left(\begin{array}{c}
-P_{1}^{\alpha} \sin \Omega^{\alpha} t \\
P_{1}^{\alpha} \cos \Omega^{\alpha} t \\
0
\end{array}\right), \quad \mathbf{p}_{2}^{\alpha}=\left(\begin{array}{c}
P_{2}^{\alpha} \sin \Omega^{\alpha} t \\
-P_{2}^{\alpha} \cos \Omega^{\alpha} t \\
0
\end{array}\right)
\end{array}\right\},
$$

Фазовым траекториям $\Lambda_{\gamma}^{\alpha}(L)$ отвечают орбиты коррелированного движения электронов по равновесным окружностям в плоскости, ортогональной вектору полного углового момента. Угловая частота движения электронов

$$
\Omega^{\alpha}=\delta \frac{P_{1}^{\alpha}}{R_{1}^{\alpha}}=\delta \frac{P_{2}^{\alpha}}{R_{2}^{\alpha}}, \quad \delta=\frac{a_{0}}{p_{0} t_{0}}, \quad a_{0}=\frac{\hbar^{2}}{m_{e} e_{0}^{2}}, \quad p_{0}=\frac{m_{e} e_{0}^{2}}{\hbar}, \quad t_{0}=\frac{\hbar^{3}}{m_{e} e_{0}^{4}},
$$

а радиусы равновесных окружностей $R_{1}^{\alpha}, R_{2}^{\alpha}$ и соответствующие импульсы электронов $P_{1}^{\alpha}, P_{2}^{\alpha}$ определяются соотношениями:

a) при $\alpha=1$ для симметричного ОПР $\Lambda_{\gamma}^{1}-$

$$
\begin{aligned}
& R_{1}^{1}=R_{2}^{1}=R^{1}=a_{0} \frac{\tilde{L}^{2}+\sqrt{\tilde{L}^{4}+48 \tilde{\gamma}(4 Z-1)}}{8 Z-2}, \\
& P_{1}^{1}=P_{2}^{1}=P^{1}=p_{0}\left(\frac{(4 Z-1) a_{0}}{4 R^{1}}-\frac{3 \tilde{\gamma} a_{0}^{3}}{\left(R^{1}\right)^{3}}\right)^{\frac{1}{2}} ;
\end{aligned}
$$

б) для ОПР $\Lambda_{\gamma}^{\alpha}(L)$ при $\alpha>1$ импульсы $P_{i}^{\alpha}$ и радиусы орбит $R_{i}^{\alpha}, i=1,2$, электронов однозначно определяются через решения алгебраического уравнения относительно параметра $k \in \mathbb{R}^{+}$,

$$
\begin{gathered}
-\frac{\tilde{L}^{2}}{\sqrt{3 \tilde{\gamma}}} \frac{1+k}{k} \sqrt{\frac{k^{4}+k^{3}+k^{2}+k+1}{Z k^{4}+3 Z k^{3}+(4 Z-1) k^{2}+3 Z k+Z}}- \\
-2 k^{7}-2 k^{6}-k^{5}+k^{4}-k^{3}+1+ \\
+\frac{1}{Z k^{4}+3 Z k^{3}+(4 Z-1) k^{2}+3 Z k+Z}\left(k^{4}+k^{3}+k^{2}+k+1\right)(1+k)^{2} \times \\
\times\left(Z k^{2}\left(1-k^{3}\right)-Z\left(1-k^{3}\right)+Z k^{3}(1+k)^{2}-k^{3}\right)=0, \\
R_{1}^{\alpha}=a_{0} \frac{3 \gamma\left(k^{4}+k^{3}+k^{2}+k+1\right)(1+k)^{2}}{k^{2}\left[Z(1+k)^{2}\left(k^{2}+k+1\right)-k^{2}\right]}, R_{2}^{\alpha}=k R_{1}^{\alpha}, \\
P_{1}^{\alpha}=p_{0}\left[\frac{Z a_{0}}{R_{1}^{\alpha}}-\frac{3 \gamma a_{0}^{3}}{\left(R_{1}^{\alpha}\right)^{3}}-\frac{R_{1}^{\alpha} a_{0}^{2}}{\left(R_{1}^{\alpha}-R_{2}^{\alpha}\right)^{2}}\right]^{\frac{1}{2}}, \\
P_{2}^{\alpha}=p_{0}\left[\frac{Z a_{0}}{R_{2}^{\alpha}}-\frac{3 \gamma a_{0}^{3}}{\left(R_{2}^{\alpha}\right)^{3}}-\frac{R_{2}^{\alpha} a_{0}^{2}}{\left(R_{1}^{\alpha}-R_{2}^{\alpha}\right)^{2}}\right]^{\frac{1}{2}}, \\
\tilde{\gamma}=\frac{\gamma}{a_{0}^{2}}, \quad \tilde{L}=\frac{L}{a_{0} p_{0}} .
\end{gathered}
$$


Численный анализ показал, что в области параметров $\tilde{\gamma}>C \tilde{L}^{4}, C \approx 0.0629$ уравнение $(17)$ имеет два решения $k_{i} \neq 1, i=2,3, k_{i} \in \mathbb{R}^{+}$. Отсюда следует полная классификация ОПР классической системы (3).

УТВЕРЖДЕНИЕ 3. Траектории $\Lambda_{\gamma}^{\alpha}(L), \alpha=1,2,3$, определенные формулами (15)(18), описывают все ОПР классической системы (3), причем:

а) в области параметров $\tilde{\gamma}>C \tilde{L}_{4}, \quad C \approx 0.0629$ существуют три ОПР $(N(\gamma, L)=3)$, при әтом $R_{1}^{\alpha}<R^{1}<R_{2}^{\alpha}, \quad \alpha=2,3 ;$ соответствующие $\alpha=2,3$ ОПР будем называть несимметричнымми

б) в области параметров $0 \leqslant \tilde{\gamma} \leqslant C \tilde{L}_{4}$ существует лишь одно симметричное ОПР $\Lambda_{\gamma}^{1}(L) \quad(N(\gamma, L)=1)$.

Нетривиальные показатели Флоке $\beta_{1,2}^{\alpha}$ матрицы (14) определяются формулами

$$
\frac{\beta_{1}^{\alpha}}{2 \pi}= \pm \sqrt{\frac{-d_{1}^{\alpha} b_{1}^{\alpha}-c_{1}^{\alpha}}{b_{2}^{\alpha}}}, \quad \frac{\beta_{2}^{\alpha}}{2 \pi}= \pm \sqrt{\frac{d_{2}^{\alpha} b_{2}^{\alpha}-c_{2}^{\alpha} b_{1}^{\alpha}}{b_{1}^{\alpha}}}
$$

где

$$
\begin{aligned}
b_{j}^{\alpha} & =\frac{3 \tilde{\gamma} a_{0}^{5}}{\left(R_{j}^{\alpha}\right)^{5}}-\frac{Z a_{0}^{3}}{\left(R_{j}^{\alpha}\right)^{3}}+\left(g^{\alpha}\right)^{3}, & c_{j}^{\alpha} & =\frac{Z\left(R_{j}^{\alpha}\right)^{2} a_{0}^{3}-9 \tilde{\gamma} a_{0}^{5}}{\left(R_{j}^{\alpha}\right)^{5}}+d_{j}^{\alpha}+2\left(g^{\alpha}\right)^{3}, \\
d_{j}^{\alpha} & =-\frac{3 R_{j}^{\alpha}}{a_{0}}\left(g^{\alpha}\right)^{4}, & g^{\alpha} & =\frac{a_{0}}{R_{1}^{\alpha}+R_{2}^{\alpha}}, \quad j=1,2 .
\end{aligned}
$$

\section{3. КВАЗИКЛАССИЧЕСКИЕ СПЕКТРАЛЬНЫЕ СЕРИИ ОПЕРАТОРА ПАУЛИ В ОТСУТСТВИЕ ВНЕШНЕГО ПОЛЯ}

3.1. Нерелятивистский гелиеподобный атом с квадрупольным моментом. Сверхтонкое расщепление. Здесь мы приведем явные формулы квазиклассических спектральных серий для задачи (1), (2), соответствующих ОПР (15). Следуя схеме квантования, описанной выше, найдем решения спектральных задач для скалярного оператора $\widehat{H}_{0}(6)$ и уравнения поляризации (7). Используя эти результаты, мы затем получим ответ в исходной задаче.

Условие квантования для семейства точек покоя $\mu_{0}^{\alpha}(L)$ с комплексным ростком $r\left(\mu_{0}^{\alpha}(L)\right)$ относительно $H_{0}=H_{0}(\mu)$ в дуальном пространстве алгебры Ли $G_{\mu}[40]$ в исходном фазовом пространстве с учетом формул (15)-(18) имеет вид

$$
\begin{gathered}
\frac{1}{2 \pi \hbar} \oint_{\Lambda_{\gamma}^{\alpha}(L)} p d q=L_{l, \nu_{1}, \nu_{2}}^{\alpha}=l+\sum_{j=1}^{2} \beta_{j}^{\alpha}(L, \gamma)\left(\nu_{j}+\frac{1}{2}\right), \\
l \in \mathbb{Z}, \quad \nu_{j} \in \mathbb{Z}^{+}, \quad \nu_{j} \ll l, \quad j=1,2 .
\end{gathered}
$$

Показатели Флоке $\beta_{j}^{\alpha}(L, \gamma)$ определены в (19). Заметим, что здесь орбитальное квантовое число $l=l(\hbar)$ есть большой параметр, $|l| \gg 1$. Он связан с параметром $\hbar(\hbar \rightarrow 0)$ условием $l(\hbar) \hbar \rightarrow L^{\text {cl }}$, где полный орбитальный момент двух частиц $L^{\text {cl }}$ определяется по значению энергии $E^{\mathrm{cl}}$ классической системы соотношениями $E^{\mathrm{cl}}=f^{\alpha}\left(L^{\mathrm{cl}}, \gamma\right)$. Для семейства $\Lambda_{\gamma}^{1}(L)$ эта зависимость имеет вид

$$
f^{1}\left(L^{\mathrm{cl}}, \gamma\right)=-\frac{(4 Z-1) e_{0}^{2}}{4 R^{1}(L)}-\frac{e_{0}^{2} \gamma}{\left(R^{1}(L)\right)^{3}} .
$$

5 Теоретическая и математическая физика, т. 126, № 3, 2001 г. 


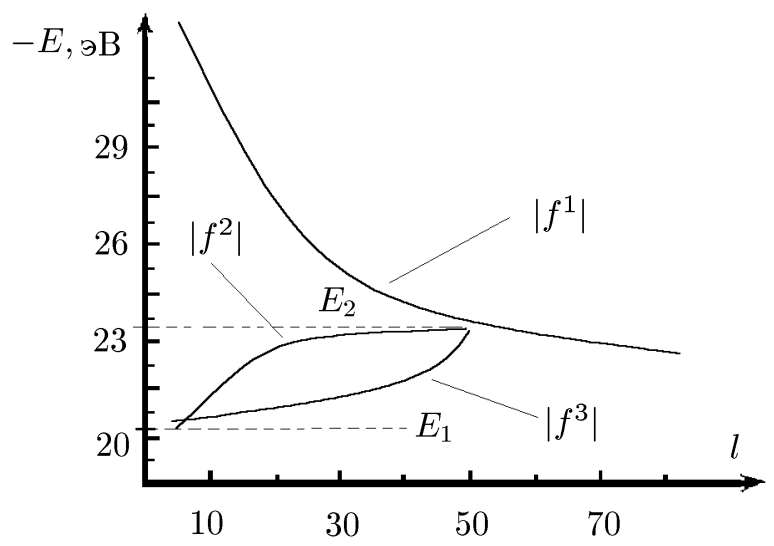

Для значений $\alpha=2,3$ на рисунке приведен лишш качественный характер зависимостей $f^{\alpha}\left(L^{\mathrm{cl}}, \gamma\right)$ при фиксированных значениях параметров $\gamma=10^{-25} \mathrm{~cm}^{2}, Z=2$.

Условие квантования ОПР (20) определяет последовательность (квазикласических) квантованных значений полного углового момента двух электронов $L^{\alpha}=L_{l, \nu_{1}, \nu_{2}}^{\alpha}(\hbar)$. Тогда квазиклассические уровни энергии скалярного оператора $\widehat{H}_{0}$, отвечающие (в пределе $\hbar \rightarrow 0$ ) семейству замкнутых фазовых кривых $\Lambda_{\gamma}^{\alpha}(L), \alpha=1,2,3$, определяются формулой

$$
E^{\alpha}=E_{l, \nu_{1}, \nu_{2}}^{\alpha}(\hbar)=E_{l}^{\alpha(0)}(\hbar)+\hbar E_{l, \nu_{1}, \nu_{2}}^{\alpha(1)}(\hbar)+O\left(\hbar^{2}\right)
$$

где $E_{l}^{\alpha(0)}$ - энергия электронов на равновесной орбите $\Lambda_{\gamma}^{\alpha}(L)$,

$$
E_{l}^{\alpha(0)}=f^{\alpha}\left(L^{\alpha}=l \hbar, \gamma\right)
$$

a $\hbar E_{l, \nu_{1}, \nu_{2}}^{\alpha(1)}(\hbar)$ - энергия малых радиальных осцилляций электронов с частотами $\omega_{j}^{\alpha}(L, \gamma)=\Omega^{\alpha} \beta_{j}^{\alpha}(L, \gamma) / 2 \pi$ при $L=l \hbar$,

$$
\hbar E_{l, \nu_{1}, \nu_{2}}^{\alpha(1)}(\hbar)=\hbar \sum_{j=1}^{2} \omega_{j}^{\alpha}(L, \gamma)\left(\nu_{j}+\frac{1}{2}\right),
$$

где $L=l \hbar, l \in \mathbb{Z}, \nu_{j} \in \mathbb{Z}^{+}$. Напомним, что при $\alpha=1$ квазиклассическая спектральная серия (21) отвечает симметричному, а при $\alpha=2,3$ - несимметричному дважды возбужденному состоянию электронов.

Из полученных формул нетрудно найти сверхтонкое расшепление квазиклассических уровней энергии, отвечаюших симметричным дважды возбужденным состояниям:

$$
\left|E_{l, \nu_{1}, \nu_{2}}^{0}(\gamma)-E_{l, \nu_{1}, \nu_{2}}^{0}(0)\right| \sim \gamma
$$

Отметим, что несимметричные дважды возбужденные состояния, порожденные несимметричными ОПР классической системы (см п. “а" утверждения 3), существуют при реальных значениях $Z=2, \gamma \sim 10^{-25} \mathrm{~cm}^{2}$ лишь в достаточно узкой области энергий $E_{1} \leqslant-E \leqslant E_{2}, \Delta E=E_{2}-E_{1} \approx 3$ эВ вблизи основного уровня $E_{0}=-24.58$ эВ, $E_{2} \approx$ 23 эВ при значениях орбитального квантового числа $l \sim 10 \div 10^{2}$. 
3.2. Тонкая структура уровней (спиновые поправки). Матрица поляризации для семейства $\Lambda_{\gamma}^{\alpha}(L)$ при $\alpha=1,2,3$ есть матрица с постоянными коэффициентами

$$
\begin{gathered}
\left.\Pi\right|_{\Lambda_{\gamma}^{\alpha}(L)}=\Pi_{\alpha}^{\mathrm{s}-\mathrm{o}}+\Pi_{\alpha}^{\mathrm{s}-\mathrm{s}}, \\
\Pi_{\alpha}^{\mathrm{s}-\mathrm{o}}=C_{1}^{\alpha} \sigma_{1}^{3}+C_{2}^{\alpha} \sigma_{2}^{3}, \\
\Pi_{\alpha}^{\mathrm{s}-\mathrm{s}}=\hbar D^{\alpha}\left(\sigma_{1}^{3} \sigma_{2}^{3}-2 \sigma_{1}^{1} \sigma_{2}^{1}-2 \sigma_{1}^{2} \sigma_{2}^{2}-3 \sigma_{1}^{2} \sigma_{2}^{1}-3 \sigma_{1}^{1} \sigma_{2}^{2}\right),
\end{gathered}
$$

где в части матрищы поляризации $\Pi_{\alpha}^{\mathrm{s}-o}$, отвечаюшей спин-орбитальному взаимодействию, константы $C_{i}^{\alpha}, i=1,2$, имеют вид

$$
\begin{aligned}
& C_{1}^{\alpha}=C_{1}^{\alpha}(L, \gamma)=\frac{e_{0}^{2}}{4 m_{e}^{2} c^{2}}\left[\left(-\frac{Z}{\left(R_{1}^{\alpha}\right)^{2}}+\frac{1}{\left(R_{1}^{\alpha}+R_{2}^{\alpha}\right)^{2}}+\frac{3 \gamma}{\left(R_{1}^{\alpha}\right)^{4}}\right) P_{1}^{\alpha}+\frac{2}{\left(R_{1}^{\alpha}+R_{2}^{\alpha}\right)^{2}} P_{2}^{\alpha}\right], \\
& C_{2}^{\alpha}=C_{2}^{\alpha}(L, \gamma)=\frac{e_{0}^{2}}{4 m_{e}^{2} c^{2}}\left[\left(-\frac{Z}{\left(R_{2}^{\alpha}\right)^{2}}+\frac{1}{\left(R_{1}^{\alpha}+R_{2}^{\alpha}\right)^{2}}+\frac{3 \gamma}{\left(R_{2}^{\alpha}\right)^{4}}\right) P_{2}^{\alpha}+\frac{2}{\left(R_{1}^{\alpha}+R_{2}^{\alpha}\right)^{2}} P_{1}^{\alpha}\right],
\end{aligned}
$$

а в спин-спиновой части $\Pi_{\alpha}^{\mathrm{s}-\mathrm{s}}$ матрицы поляризации константа

$$
D^{\alpha}=D^{\alpha}(L, \gamma)=\frac{e_{0}^{2}}{2 m_{e}^{2} c^{2}} \frac{1}{\left(R_{1}^{\alpha}+R_{2}^{\alpha}\right)^{3}} .
$$

В дальнейшем для сокрашения обозначений индекс $\alpha$ в константах $C_{i}^{\alpha}, D^{\alpha}$ опушен.

Поскольку оператор дифференцирования вдоль кривой $\Lambda_{\gamma}^{\alpha}(L)$

$$
\left.\frac{d}{d t}\right|_{\Lambda_{\gamma}^{\alpha}(L)}=2 \Omega^{\alpha} \frac{\partial}{\partial \varphi} \quad\left(\varphi=\varphi_{1}=\varphi_{2}\right)
$$

спектральная задача (7) принимает вид

$$
\left(-2 i \Omega^{\alpha} \frac{\partial}{\partial \varphi}+\left.\Pi\right|_{\Lambda_{\gamma}^{\alpha}(L)}\right) \mathbf{f}_{\xi}=\mu_{\xi} \mathbf{f}_{\xi}, \quad \mathbf{f}_{\xi}(\varphi+2 \pi)=\mathbf{f}_{\xi}(\varphi), \quad \alpha=1,2,3, \quad \mathbf{f}(\xi) \in \mathbb{C}^{4}
$$

Ее решения определяются следуюшими формулами $(k \in \mathbb{Z})$ :

a) $\quad \xi= \pm 1: \quad \mathbf{f}_{\xi}(\varphi)=e^{i k \varphi} v_{\xi}, \quad \mu_{ \pm 1}=2 \Omega^{\alpha} k \pm \sqrt{\left(C_{1}+C_{2}\right)^{2}+36 \hbar^{2} D^{2}}+\hbar D$

$$
\begin{aligned}
& v_{+1}={ }^{t}(1,0,0, F(\hbar)), \quad v_{-1}={ }^{t}(F(\hbar), 0,0,1), \\
& F(\hbar)=\frac{-C_{1}-C_{2}-\sqrt{\left(C_{1}+C_{2}\right)^{2}+36 \hbar^{2} D^{2}}}{6 \hbar D i} ;
\end{aligned}
$$

б) $\xi=0: \quad \mathbf{f}_{\xi}^{(m)}(\varphi)=e^{i k \varphi} v_{0}^{(m)}$,

$$
\mu_{0}^{(m)}=2 \Omega^{\alpha} k-(-1)^{m} \sqrt{\left(C_{1}-C_{2}\right)^{2}+16 \hbar^{2} D^{2}}-\hbar D,
$$

где $m=1,2$ - номер вырожденного собственного значения $\xi=0$ спектральной задачи (22), отвечаюшего нулевому значению проекции спина электронов на ось $z: m_{s}=0$ с полным спином $S=1$ для $m=1$ и $S=0$ для $m=2$, соответственно,

$$
\begin{aligned}
v_{0}^{(1)} & ={ }^{t}(0,-1, G(\hbar), 0), \quad v_{0}^{(2)}={ }^{t}(0, G(\hbar), 1,0), \\
G(\hbar) & =\frac{C_{2}-C_{1}+\sqrt{\left(C_{1}-C_{2}\right)^{2}+16 \hbar^{2} D^{2}}}{4 \hbar D} .
\end{aligned}
$$


Без потери обшности можно положить здесь $k=0$, так как случай $k \neq 0$ приводит лиш к перенумерации квазиклассических уровней энергии (21) скалярной задачи в силу равенства $f^{\alpha}(l \hbar, \gamma)+2 \hbar \Omega^{\alpha} k+O\left(\hbar^{2}\right)=f^{\alpha}((l+k) \hbar, \gamma)$. Из этих формул нетрудно получить с точностью до $O\left(\hbar^{3}\right)$ спиновые поправки $\Delta E=\Delta E^{\mathrm{s}-\mathrm{o}}+\Delta E^{\mathrm{s}-\mathrm{s}}=\hbar \mu_{\xi}$ к квазиклассическим уровням энергии $E^{\alpha}(\alpha=1,2,3)(21)$, где $\Delta E^{\text {s-o }}$ - энергия спин-орбитального взаимодействия, а $\Delta E^{\mathrm{S}-\mathrm{s}}$ - энергия взаимодействия спинов 2 -х электронов:

a) $\xi= \pm 1, \quad \alpha=1,2,3: \Delta E^{\mathrm{s}-\mathrm{o}}=\xi \hbar\left(C_{1}+C_{2}\right), \quad \Delta E^{\mathrm{s}-\mathrm{s}}=\hbar^{2} D$

б) $\xi=0, \quad \alpha=2,3: \quad \Delta E^{\mathrm{s}-\mathrm{o}(m)}=(-1)^{m} \hbar\left(C_{2}-C_{1}\right), \quad \Delta E^{\mathrm{S}-\mathrm{s}(m)}=\hbar^{2} D$,

в) $\xi=0, \quad \alpha=1: \quad \Delta E^{\mathrm{s}-\mathrm{o}(m)}=0, \quad \Delta E^{\mathrm{s}-\mathrm{s}(m)}=\Xi^{(m)} \hbar^{2} D$,

$$
\Xi^{(1)}=3, \quad \Xi^{(2)}=-5 \text {. }
$$

Тогда квазиклассические спектральные серии для исходной задачи (1), (2), отвечающие $\Lambda_{\gamma}^{\alpha}(L)$, имеют вид

$$
\begin{aligned}
E_{l, \nu_{1}, \nu_{2}, \pm 1}^{\alpha}(\hbar)= & f^{\alpha}(l \hbar, \gamma)+\hbar\left[\sum_{j=1}^{2} \omega_{j}^{\alpha}(l \hbar, \gamma)\left(\nu_{j}+\frac{1}{2}\right) \pm\left(C_{1}+C_{2}\right)\right]+\hbar^{2} D+O\left(\hbar^{3}\right) \\
E_{l, \nu_{1}, \nu_{2}, 0}^{\alpha(m)}(\hbar)= & f^{\alpha}(l \hbar, \gamma)+\hbar\left[\sum_{j=1}^{2} \omega_{j}^{\alpha}(l \hbar, \gamma)\left(\nu_{j}+\frac{1}{2}\right)-\right. \\
& \left.-(-1)^{m}\left(C_{1}-C_{2}\right)\right]-\hbar^{2} D+O\left(\hbar^{3}\right), \quad \alpha=2,3, \quad m=1,2 \\
E_{l, \nu_{1}, \nu_{2}, 0}^{\alpha(m)}(\hbar)= & f^{\alpha}(l \hbar, \gamma)+\hbar\left[\sum_{j=1}^{2} \omega_{j}^{\alpha}(l \hbar, \gamma)\left(\nu_{j}+\frac{1}{2}\right)\right]+ \\
& +\Xi^{(m)} \hbar^{2} D+O\left(\hbar^{3}\right), \quad \alpha=1, \quad m=1,2 .
\end{aligned}
$$

Из полученных формул найдем тонкую структуру квазиклассических уровней (21) энергии гелиеподобного атома в симметричном $(\alpha=1)$ и несимметричных $(\alpha=2,3)$ дважды возбужденных квазиклассических состояниях. В частности, из (23) для симметричных дважды возбужденных состояний при $\gamma=0$ получаем формулу тонкого расшепления $\Delta E^{\mathrm{S}-\mathrm{o}}$ квазиклассических уровней (21), связанного со спин-орбитальным взаимодействием (в ридберговских единищах) в следуюшем виде:

$$
\Delta E_{l, \nu_{1}, \nu_{2}, \xi}^{\mathrm{s}-\mathrm{o}}=\xi \frac{\alpha^{2}}{32} \frac{(3-4 Z)(4 Z-1)^{3}}{l^{5}}
$$

где $\alpha$ - постоянная тонкой структуры $\left(\alpha=e_{0}^{2} / \hbar c\right), \xi= \pm 1, l \gg 1$. Эта квазиклассическая формула по порядку величины расщепления совпадает с известным результатом [32] (см. формулу (40.6) в этой книге), где методом теории возмушений получено тонкое расщепление для гелиеподобного иона с произвольным $Z$, когда по крайней мере один внешний электрон находится в сильно возбужденном состоянии. Отличие состоит в несколько иной зависимости величины расшепления от заряда ядра $Z$. 
Для этих же состояний величина расщепления, связанная со спин-спиновым взаимодействием электронов (в ридберговских единицах) имеет вид

$$
\begin{gathered}
\Delta E_{l, \nu_{1}, \nu_{2}, \xi}^{\mathrm{s}-\mathrm{s}}=S(\xi) \frac{\alpha^{2}}{16} \frac{(4 Z-1)^{3}}{l^{6}}, \\
\xi= \pm 1,0, \quad S( \pm 1)=1, \quad S(0)=3 \text { при } m=1, \quad S(0)=-5 \text { при } m=2 .
\end{gathered}
$$

\section{4. КВАЗИКЛАССИЧЕСКИЕ СПЕКТРАЛЬНЫЕ СЕРИИ ОПЕРАТОРА ПАУЛИ ВО ВНЕШНЕМ ПОЛЕ}

Вычислим спектральные серии гамильтониана $\widehat{H}(2)$, когда внешнее однородное магнитное поле $B_{0} \neq 0$ (без учета квадрупольного момента ядра атома гелия, $\gamma=0$ ). В этом случае процедура редукции по диагональному действию группы $S O(2)$ на пространстве $\mathbb{R}_{\mathbf{x}, \mathbf{y}}^{4} \times \mathbb{R}_{\mathbf{p}_{\mathbf{x}}, \mathbf{p}_{\mathbf{y}}}^{4} \times\left\{z_{1}=z_{2}=p_{z_{1}}=p_{z_{2}}=0\right\}$ аналогична общей схеме редукции, описанной в разделе 2 , и приводит к следуюшему результату. Сушествует лишь единственное симметричное ОПР $\Lambda_{B}(L)$, определяемое формулой $(15)$, в которой частота движения электронов

$$
\begin{gathered}
\Omega^{\alpha}=\Omega=\frac{1}{t_{0}}\left(\frac{P}{R} \frac{a_{0}}{p_{0}}+\frac{\widetilde{B}_{0}}{2}\right), \\
a_{0}=\frac{\hbar^{2}}{m_{e} e_{0}^{2}}, \quad p_{0}=\frac{m_{e} e_{0}^{2}}{\hbar}, \quad t_{0}=\frac{\hbar^{3}}{m_{e} e_{0}^{4}}, \quad \widetilde{B}_{0}=\frac{B_{0} e_{0} a_{0}^{2}}{\hbar},
\end{gathered}
$$

импульс электронов на равновесной орбите

$$
P_{1}^{\alpha}=P_{2}^{\alpha}=P=\sqrt{\frac{(4 Z-1) a_{0}^{3}+\widetilde{B}_{0}^{2} R^{3}}{4 R a_{0}^{2}}} p_{0},
$$

а радиус равновесной окружности $R_{1}^{\alpha}=R_{2}^{\alpha}=R$ определяется из алгебраического уравнения

$$
\frac{B_{0}^{2} e_{0}^{2} R^{4}}{c^{2}}+m_{e} e_{0}^{2} R(4 Z-1)-L^{2}=0, \quad L>0 .
$$

Условие квантования ОПР $\Lambda_{B}(L)$, так же как и в случае $B_{0}=0, \gamma \neq 0$, имеет вид (20). Квазиклассические уровни энергии скалярного оператора $\widehat{H}_{0}$, отвечаюшие (в пределе $\hbar \rightarrow 0$ ) семейству замкнутых фазовых кривых $\Lambda_{B}(L)$, определяются формулой

$$
E=E_{l, \nu_{1}, \nu_{2}}(\hbar)=E_{l}^{(0)}(\hbar)+\hbar E_{l, \nu_{1}, \nu_{2}}^{(1)}(\hbar)+O\left(\hbar^{2}\right)
$$

где $E_{l}^{(0)}$ - энергия электронов на равновесной орбите $\Lambda_{B}(L)$,

$$
E_{l}^{(0)}=f(L=l \hbar)=-\frac{(4 Z-1) e_{0}^{2}}{4 R}+\frac{e_{0} B_{0} L}{2 m_{e}}+\frac{e_{0}^{2} B_{0}^{2} R^{2}}{2 m_{e}},
$$

a $\hbar E_{l, \nu_{1}, \nu_{2}}^{(1)}(\hbar)$ - энергия малых радиальных осцилляций электронов с частотами $\omega_{j}(L)=$ $\Omega \beta_{j}(L, 0) / 2 \pi$ при $L=l \hbar$ (показатели Флоке $\beta_{j}(L, 0)=\beta_{j}(R(L), 0)$ определены в (19) при $\gamma=0)$,

$$
\hbar E_{l, \nu_{1}, \nu_{2}}^{(1)}(\hbar)=\hbar \sum_{j=1}^{2} \omega_{j}(l \hbar)\left(\nu_{j}+\frac{1}{2}\right), \quad \nu_{j} \in \mathbb{Z}^{+} .
$$


Матрица поляризации для семейства $\Lambda_{B}(L)$ есть матрица с постоянньми коэффициентами следуюшего вида:

$$
\left.\Pi\right|_{\Lambda_{B}(L)}=C\left(\sigma_{1}^{3}+\sigma_{2}^{3}\right)+\hbar D\left(\sigma_{1}^{3} \sigma_{2}^{3}-2 \sigma_{1}^{1} \sigma_{2}^{1}-2 \sigma_{1}^{2} \sigma_{2}^{2}-3 \sigma_{1}^{2} \sigma_{2}^{1}-3 \sigma_{1}^{1} \sigma_{2}^{2}\right),
$$

здесь

$$
\begin{gathered}
C=C(L)=\frac{e_{0}}{m_{e} c} B_{0}+\frac{e_{0}^{2}}{2 m_{e}^{2} c^{2}} \frac{3-4 Z}{4 R^{2}} P, \\
D=D(L)=\frac{e_{0}^{2}}{16 m_{e}^{2} c^{2}} \frac{1}{R^{3}} .
\end{gathered}
$$

Решения соответствующей спектральной задачи (7) для спинора $\mathbf{f}_{\xi}$ определяются формулами:

a) $\quad \xi= \pm 1: \quad \mathbf{f}_{\xi}(\varphi)=e^{i k \varphi} v_{\xi}, \quad \mu_{ \pm 1}=2 \Omega k \pm \sqrt{4 C^{2}+36 \hbar^{2} D^{2}}+\hbar D$,

$$
\begin{aligned}
& v_{+1}={ }^{t}(1,0,0, F(\hbar)), \quad v_{-1}={ }^{t}(F(\hbar), 0,0,1), \\
& F(\hbar)=\frac{-2 C-\sqrt{4 C^{2}+36 \hbar^{2} D^{2}}}{6 \hbar D i}
\end{aligned}
$$

б) $\xi=0: \quad \mathbf{f}_{\xi}^{(m)}(\varphi)=e^{i k \varphi} v_{0}^{(m)}, \quad \mu_{\xi}^{(m)}=2 \Omega^{\alpha} k+\Xi^{(m)} \hbar^{2} D+O\left(\hbar^{3}\right)$,

где $m=1,2$ - номер вырожденного собственного значения $\xi=0$ спектральной задачи, отвечающего значению спинового квантового числа $m_{s}=0$ с полным спином $S=1$ и $S=0$, соответственно,

$$
v_{0}^{(1)}={ }^{t}(0,-1,1,0), \quad v_{0}^{(2)}={ }^{t}(0,1,1,0), \quad \Xi^{(1)}=3, \quad \Xi^{(2)}=-5, \quad k \in \mathbb{Z} .
$$

В п. б, так же, как в п. 3.2, можно положить $k=0$. Тогда спиновые поправки $\Delta E=$ $\Delta E^{\mathrm{s}-\mathrm{o}}+\Delta E^{\mathrm{s}-\mathrm{f}}+\Delta E^{s-s}=\hbar \mu_{\xi}$ к квазиклассическим уровням энергии $(25)$ с точностью $O\left(\hbar^{3}\right)$ имеют вид:

$$
\begin{array}{ll}
\text { а) } \quad \xi= \pm 1: & \Delta E^{\mathrm{s}-0}+\Delta E^{\mathrm{s}-\mathrm{f}}=2 \xi \hbar C, \quad \Delta E^{\mathrm{s}-\mathrm{s}}=\hbar^{2} D ; \\
\text { б) } \quad \xi=0: \quad \Delta E^{\mathrm{s}-\mathrm{o}(m)}+\Delta E^{\mathrm{s}-\mathrm{f}(m)}=0, \quad \Delta E^{\mathrm{s}-\mathrm{s}(m)}=\Xi^{(m)} \hbar^{2} D, \\
& \Xi^{(1)}=3, \quad \Xi^{(2)}=-5 .
\end{array}
$$

Здесь $m=1,2 ; \Delta E^{\mathrm{s}-\mathrm{o}}$ - энергия спин-орбитального взаимодействия, $\Delta E^{\mathrm{s}-\mathrm{f}}-$ энергия взаимодействия внешнего магнитного поля со спинами электронов, а $\Delta E^{\mathrm{s}-\mathrm{s}}-$ энергия спин-спинового взаимодействия двух электронов.

Тогда квазиклассические спектральные серии исходной задачи (1), (2), порожденные семейством $\Lambda_{B}(L)$, задаются формулами

$$
\begin{aligned}
E_{l, \nu_{1}, \nu_{2}, \pm 1}(\hbar) & =f(l \hbar)+\hbar\left[\sum_{j=1}^{2} \omega_{j}(l \hbar)\left(\nu_{j}+\frac{1}{2}\right) \pm 2 C\right]+\hbar^{2} D+O\left(\hbar^{3}\right), \\
E_{l, \nu_{1}, \nu_{2}, 0}^{(m)}(\hbar) & =f(l \hbar)+\hbar \sum_{j=1}^{2} \omega_{j}(l \hbar)\left(\nu_{j}+\frac{1}{2}\right)+\Xi^{(m)} \hbar^{2} D+O\left(\hbar^{3}\right) .
\end{aligned}
$$


Полученные формулы позволяют вычислить расщепление квазиклассических уровней энергии гелиеподобного иона с произвольным зарядом ядра $Z$ под влиянием внешнего магнитного поля - эффект Зеемана с учетом диамагнетизма атома (нормальный эффект Зеемана - расшепление уровней $\delta E^{\mathrm{o}-\mathrm{f}}$ в результате взаимодействия поля с орбитальным магнитным моментом электронов и аномальный эффект Зеемана - расщепление уровней $\delta E^{\mathrm{s}-\mathrm{f}}$ за счет взаимодействия поля со спинами электронов) при любом значении напряженности поля $B_{0}$, включая промежуточные поля $B_{0} \sim 10^{9}$ Гс. Именно эта область полей (в которой кулоновское и магнитное взаимодействия одного порядка) наиболее трудна для получения аналитических результатов, в частности, методами теории возмущений (см., например, работу [27], (и имеющуюся в ней библиографию), в которой для полей в промежуточной области дан численный расчет нормального зеемановского расшепления на основе модификации метода Хартри-Фока с помощью $B$-сплайн функций). В нашем случае получение численных значений зеемановского расшепления $\delta E=\delta E^{\mathrm{o}-\mathrm{f}}+\delta E^{\mathrm{s}-\mathrm{f}}$ сводится к численному решению лишш одного алгебраического уравнения (24). Ниже в пп. 4.1, 4.2 получены явные (аналитические) формулы зеемановского расщепления в предельных случаях слабого $\left(B_{0} \rightarrow 0\right)$ и сильного $\left(B_{0} \rightarrow \infty\right)$ магнитных полей.

4.1. Эффект Зеемана в приближении слабого поля. По аналогии с одночастичной задачей для атома водорода в магнитном поле [33] введем ларморовский радиус $a_{H}=\left(c \hbar / e_{0} B_{0}\right)^{1 / 2}$ и определим слабое магнитное поле в терминах отношения $\left(a_{0} / a_{H}\right)^{4}=\mathcal{E}_{w}$, где $a_{0}=\hbar^{2} /\left(m_{e} e_{0}^{2}\right)$ - боровский радиус электрона. Параметр $\varepsilon_{w}=$ $\mathcal{E}_{w} /(4 Z-1)^{3}$ эффективно мал для значений $B_{0}$ вплоть до $10^{3} \Gamma$ с. В этом случае найдем приближенное значение радиуса $R$ из уравнения $(24)$ при $\varepsilon_{w} \rightarrow 0$, полагая $L=l \hbar, l \neq 0$. Реальным параметром разложения в этом случае является параметр

тогда

$$
q_{w}=\varepsilon_{w} l^{6}=\left(\frac{l^{3} B_{0} \hbar^{3}}{c m_{e}^{2} e_{0}^{3}}\right)^{2}(4 Z-1)^{-3} \ll 1,
$$

$$
R(L=l \hbar)=\frac{\hbar^{2}}{m_{e} e_{0}^{2}} l^{2} \sum_{j=0}^{\infty} r_{j}^{w} q_{w}^{j} .
$$

В частности, $r_{0}^{w}=1, r_{1}^{w}=-1$. После подстановки (28) в (25) мы получим квазиклассические уровни энергии оператора Шредингера в магнитном поле $\widehat{H}_{0}(2)$, отвечающие замкнутой фазовой кривой $\Lambda_{B}(L)$, в приближении слабого магнитного поля $\left(B_{0} \rightarrow 0\right)$ в виде асимптотического разложения по степеням параметра $q_{w} \ll 1$,

$$
\begin{aligned}
E_{l, \nu_{1}, \nu_{2}}(\hbar)= & \frac{\hbar \omega_{0}}{l^{2}}(4 Z-1)^{2}\left[-\frac{1}{4}+\left(\nu_{1}+\frac{1}{2}\right)(4|l|)^{-1}+\left(\nu_{2}+\frac{1}{2}\right)(4|l|)^{-1}\right]+\frac{\omega_{B} \hbar l}{2}+ \\
& +\frac{\hbar \omega_{0}}{l^{2}}(4 Z-1)^{2} \sum_{j=1}^{\infty} C_{j}^{w}\left(\nu_{1}, \nu_{2}, l\right) q_{w}^{j}+O\left(\hbar^{2}\right),
\end{aligned}
$$

где $\omega_{0}=m_{e} e_{0}^{4} / \hbar^{3}$ - частота движения электрона по боровской орбите в атоме водорода, а циклотронная частота $\omega_{B}=e_{0} B_{0} / m_{e} c$. В частности, первый коэффициент разложения имеет вид

$$
C_{1}^{w}=-\frac{1}{4}-5\left(\nu_{1}+\frac{1}{2}\right)(8|l|)^{-1}-3\left(\nu_{2}+\frac{1}{2}\right)(8|l|)^{-1}
$$


Формула (29), во-первых, дает при $B_{0}=0$ квазиклассические уровни энергии гелиеподобного иона с произвольным зарядом ядра $Z$ - спектральную серию, отвечаюшую в пределе $\hbar \rightarrow 0$ коррелированному движению двух электронов по равновесной окружности радиуса $R_{0}=\hbar^{2} l^{2} /\left(m_{e} e_{0}^{2}\right), \hbar l \sim 1$, в плоскости $z=0$. С точностью до $O\left(\hbar^{2}\right)=$ $O\left((\nu / l)^{2}\right), \nu / l \ll 1$, где $\nu=\max \left(\nu_{1}, \nu_{2}\right)$, эти уровни можно представить в виде тройной ридберговской серии

$$
E_{N}(h)=-\frac{\hbar \omega_{0}(4 Z-1)^{2}}{N^{2}}
$$

с "главным" квантовым числом $N=2|l|+\nu_{1}+\nu_{2}+1$. И, во-вторых, - расщепление этих уровней в слабом магнитном поле $B_{0}$ (нормальный эффект Зеемана с учетом диамагнетизма атома) с любой степенью точности по четным степеням $B_{0}$. В частности, с точностью до $O\left(B_{0}^{4}\right), B_{0} \rightarrow 0$, это расшепление определяется формулой

$$
\delta E^{\mathrm{o}-\mathrm{f}}=\frac{\omega_{B} \hbar l}{2}+\frac{\hbar \omega_{0}}{l^{2}}(4 Z-1)^{2} C_{1}^{w} q_{w} .
$$

Подставляя разложение для радиуса (28) в (27), получим формулу для спиновых поправок в следуюшем виде:

$$
\begin{aligned}
\Delta E^{\mathrm{s}-\mathrm{o}}+\Delta E^{\mathrm{s}-\mathrm{f}}= & \xi \hbar \omega_{B}+\frac{\xi}{32} \frac{m_{e} e_{0}^{8}}{\hbar^{4} l^{5} c^{2}}(3-4 Z)(4 Z-1)^{2} \times \\
& \times\left[(4 Z-1)+\left(6 Z-\frac{3}{2}\right) q_{w}+O\left(q_{w}^{2}\right)\right] .
\end{aligned}
$$

Отсюда следует, что расщепление квазиклассических уровней энергии гелиеподобного иона с произвольным зарядом ядра $Z$, обусловленное взаимодействием спинов электронов со слабым магнитным полем (аномальный эффект Зеемана с учетом диамагнетизма атома), имеет вид

$$
\delta E^{\mathrm{s}-\mathrm{f}}=\xi \frac{e_{0} \hbar}{m_{e} c} B_{0}+\delta E_{\mathrm{diam}}^{\mathrm{s}-\mathrm{f}},
$$

где расшепление $\delta E_{\mathrm{diam}}^{\mathrm{s}-\mathrm{f}}$ (в ридберговских единицах)

$$
\delta E_{\text {diam }}^{\mathrm{s}-\mathrm{f}}=\frac{\xi}{32} \frac{\alpha^{2}}{l^{5}}(3-4 Z)(4 Z-1)^{2}\left(6 Z-\frac{3}{2}\right) q_{w}+O\left(q_{w}^{2}\right), \quad \alpha=\frac{e_{0}^{2}}{\hbar c}, \quad \xi= \pm 1 .
$$

4.2. Эффект Зеемана в приближении сильного поля. Рассмотрим сильное магнитное поле такое, что боровский радиус $a_{0}=\hbar^{2} / m_{e} e_{0}^{2}$ много больше ларморовского радиуса $a_{H}=\left(c \hbar / e_{0} B_{0}\right)^{1 / 2}$. Условие $a_{H} \ll a_{0}$ эффективно выполняется для полей начиная с $B_{0} \sim 4.7 \times 10^{9} \Gamma$ c. Параметром разложения в этом случае является параметр $q_{s}$,

$$
q_{s}=\left(\frac{m_{e}^{2} c e_{0}^{3}}{16 B_{0}}\right)^{\frac{1}{2}}(4 Z-1)(|l| \hbar)^{-\frac{3}{2}} \ll 1,
$$

тогда

$$
R(L=l \hbar)=\left(\frac{c \hbar|l|}{e_{0} B_{0}}\right)^{\frac{1}{2}} \sum_{j=0}^{\infty} r_{j}^{s} q_{s}^{j} .
$$


В частности, $r_{0}^{w}=1, r_{1}^{w}=-1 / 4$. После подстановки (31) в (25) получим квазиклассические уровни энергии оператора Шредингера в магнитном поле $\widehat{H}_{0}$, отвечающие замкнутой фазовой кривой $\Lambda_{B}(L)$, в приближении сильного магнитного поля $\left(B_{0} \rightarrow \infty\right)$ в виде разложения по степеням $q_{s}$ :

$$
E_{l, \nu_{1}, \nu_{2}}(\hbar)=\hbar \omega_{B} \sum_{j=0}^{\infty} C_{j}^{s}\left(\nu_{1}, \nu_{2}, l\right) q_{s}^{\frac{j}{2}}+O\left(\hbar^{2}\right) .
$$

Первые два коэффициента разложения имеют вид

$$
C_{0}^{s}=\nu_{1}+\nu_{2}+1+2|l|, \quad C_{1}^{s}=\frac{1}{4}\left(\nu_{1}+\nu_{2}+1\right) .
$$

Подставляя разложение для радиуса (31) в (27), получим приближенную с точностью до $O\left(1 / B_{0}\right), B_{0} \rightarrow \infty$, формулу для “аномального" расщепления уровней энергии в следуюшем виде $(\xi= \pm 1)$ :

$$
\Delta E^{\mathrm{S}-\mathrm{f}}=\xi \hbar \omega_{B}+\frac{\xi}{32} \frac{e_{0}^{\frac{5}{2}} B_{0}^{\frac{1}{2}} l}{\sqrt{2 m_{e}^{2} c^{5} \hbar|l|^{3}}}(3-4 Z)\left[(4 Z-1)+\left(\frac{3 Z}{2}-\frac{3}{8}\right) q_{w}\right] .
$$

Благодарности. Авторы выражают искреннюю благодарность И. В. Тютину за полезное обсуждение результатов работы.

\section{Список литературы}

[1] В. П. Маслов. Теория возмущений и асимптотические методы. М.: МГУ, 1965.

[2] В. П. Маслов, М. В. Федорюк. Квазиклассическое приближение для уравнений квантовой механики. М.: Наука, 1976.

[3] Ж. Лере. Лагранжев анализ и квантовая механика. Математическая структура, связанная с асимптотическими разложениями и индексом Маслова. М.: Мир, 1981.

[4] В. П. Маслов. Комплексный метод ВКБ в нелинейных уравнениях. М.: Наука, 1977.

[5] В. В. Белов, С. Ю. Доброхотов. ДАН СССР, сер. Математика. 1988. Т. 298. № 5. С. 1037.

[6] В. В. Белов, С. Ю. Доброхотов. ТМФ. 1992. Т. 92. С. 215.

[7] I. Langmuir. Phys. Rev. 1921. V. 17. P. 339.

[8] U. Fano. Rep. Prog. Phys. 1983. V. 46. P. 97.

[9] D. Wintgen, A. Bürgers, K. Richter, G. Tanner. Prog. Theor. Phys. Suppl. 1994. V. 116. P. 121.

[10] J. A. West, Z. D. Gaeta, C. R. Stroud, Jr. Phys. Rev. A. 1998. V. 58. № 1. P. 186.

[11] J. Müller, J. Burgdörfer. Phys. Rev. Lett. 1993. V. 70. № 16. P. 2375.

[12] J. Müller, X. Yang, J. Burgdörfer. Phys. Rev. A. 1994. V. 49. № 4. P. 2470.

[13] K. Richter, D. Wintgen. Phys. Rev. Lett. 1995. V. 65. № 15. P. 167.

[14] F. Benvenuto, G. Casati, D. L. Shepelyansky. Phys. Rev. A. 1996. V. 53. P. 737.

[15] U. Eichmann, V. Lange, W. Sandner. Phys. Rev. Lett. 1990. V. 64. P. 274; 1992. V. 68. P. 21.

[16] K. Richter, D. Wintgen. J. Phys. B. 1990. V. 23. P. L197.

[17] K. Richter, D. Wintgen. J. Phys. B. 1991. V. 24. P. L565.

[18] M. C. Gutzwiller. Chaos in Classical and Quantum Mechanics. N.Y.: Springer, 1990.

[19] A. Bürgers, D. Wintgen. J. Phys. B. 1994. V. 27. P. L131.

[20] J. Müller, J. Burgdörfer, D. Noid. Phys. Rev. A. 1992. V. 45. № 3. P. 1471.

[21] P. Gaspard, S. A. Rice. Phys. Rev. A. 1993. V. 48. № 1. P. 54.

[22] В. И. Арнольд. Математические методы классической механики. М.: Наука, 1983. 
[23] J. E. Marsden, A. Weinstein. Rep. Math. Phys. 1974. V. 5. P. 121.

[24] И. И. Собельман. Введение в теорию атомных спектров. М.: Физматгиз, 1968.

[25] J. Trümper, W. Pietsch, C. Reppin, W. Voges, R. Staubert, E. Kendziorra. Astrophys. J. 1978. V. 219. P. L105.

[26] R. J. Elliott, R. Louden. J. Phys. Chem. Solids. 1960. V. 15. P. 196.

[27] H. Qiao, B. Li. Phys. Rev. A. 1999. V. 60. № 4. P. 3134.

[28] H.Ögelman. The Lives of the Neutron Stars. NATO Adv. Stud. Inst. Ser. C: Math. Phys. Sci. V. 450. Eds. M.A. Alpar, Ü. Kiziloglu, J. van Paradijs. Dordrecht: Kluwer, 1995.

[29] W. Becker, J. Trümper. Astron. Astrophys. 1997. V. 326. P. 682.

[30] V. G. Bezchastnov, G. G. Pavlov, J. Ventura. Phys. Rev. A. 1998. V. 58. № 1. P. 180.

[31] A. B. Карапетян. Устойчивость стационарных движений. М.: Эдиториал УРСС, 1998.

[32] Г. Бете, Э. Солпитер. Квантовая механика атомов с одним и двумя электронами. Физматгиз, 1969.

[33] V. V. Belov, V. M. Olivè, J. L. Volkova. J. Phys. A. 1995. V. 28. P. 5799; P. 5811.

[34] В. В. Козлов. Симметрии, топология и резонансы в гамильтоновой механике. Ижевск: Изд-во УГУ, 1995.

[35] B. Valiño, S. Yu. Dobrokhotov, N. N. Nekhoroshev. УМH. 1984. T. 39. № 3. C. 233.

[36] S. Yu. Dobrokhotov, A. I. Shafarevich. Russ. J. Math. Phys. 1998. V. 5. № 2. P. 267.

[37] S. Yu. Dobrokhotov, V. M. Olivè, A. I. Shafarevich. Russ. J. Math. Phys. 1997. V. 3. № 1. P. 133.

[38] В. В. Белов, О. С. Доброхотов, С. Ю. Доброхотов. Матем. заметки. 2001. Т. 69. № 4. C. 483.

[39] А. А. Кириллов. Элементы теории представлений. М.: Наука, 1979.

[40] Ю. М. Воробъев. Квазиклассическое квантование изотропных подмногообразий в некоторых многочастичных задачах. Дисс. на соискание уч. степени канд. физ.-матем. наук. М.: МИЭМ, 1983. 